\title{
THE ENERGETICS AND DYNAMICS OF FREE RADICALS, IONS, AND CLUSTERS
}

\author{
Progress Report
}

August 1, 1991 - MARCH 31, 1992

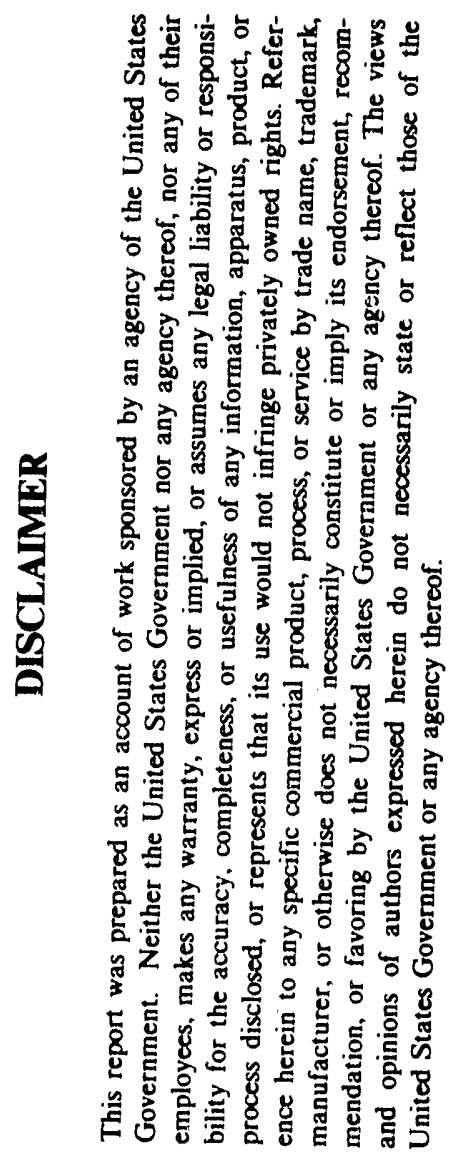

\author{
TOMAS BAER \\ Chemistry Department \\ University of North Carolima \\ Chapel Hill, NC 27599-3290
}

MARCh 1992

PREPARED FOR THE U.S. DEPARTMENT OF ENERGY UNDER GRANT NUNBER DE-FG05-88ER-13950 


\title{
THE ENERGETICS AND DYMAMICS OF FREE RADICALS, IONS, AND CLUSTERS DE-FG05-88ER 13950
}

\author{
Tomas Baer, Principal Investigator \\ Progress Report March, 1992
}

The structure and energetics of free radicals, ions, and clusters have been investigated by photoelectron photoion coincidence (PEPICO) and analyzed with ab initio molecular orbital and statistical theory RRKM calculations. In these experiments, molecules are prepared in a molecular beam so that their internal as well as translational energies are cooled to near $0 \mathrm{~K}$. The coincidence condition between energy analyzed electrons and their corresponding ions insures that the ions are energy selected. The primary experimental information includes ionization and fragment ion appearance energies, and the ion time of flight (TOF) distributions. The latter are obtained by using the energy selected electron as a start signal and the ion as the stop signal. These types of experiments allow us to measure the ion dissociation rates in the $10^{4}$ to $10^{7} \mathrm{sec}^{-1}$ range. Such ions are commonly referred to as metastable ions. In addition, the TOF peak widths are related to the release of translational energy in the ion dissociation process.

\section{Summary of Major Results:}

Perhaps the most important advance during the past year has been in the study of cluster photoionization. We have developed an experimental method for differentiating similar. mass cluster ions produced by the reactions:

$$
\begin{aligned}
& (A B n \rightarrow(A B))^{+} \\
& (A b) n+m \rightarrow(A B)_{n}^{+}+(A B)_{m}
\end{aligned}
$$

This method is based on the kinetic energy of the ions measured by TOF.

One of the major problems in the study of clusters is the determination of the size distribution. Aside from spectroscopic identification of clusters which works well for dimers and trimers, mass analysis by mass spectrometry is virtually the only method for obtaining this information. However, there is a fundamental problem in this analysis which is that cluster ions can dissociate in the ionization process and produce ionic clusters of lower mass. This is a particularly severe problem in electron impact ionization in which the electron energy is $i 11$ defined. However, the problem also exists in photoionization where the photon energy is narrow and variable. Thus, the cluster ion distribution may not reflect the neutral size distribution. The problem is particularly difficult to deal with when the ion dissociates by loosing one of the neutral molecular units, because then the $(A B)_{n}^{+}$ions from reactions (1) and (2) cannot be distinguished.

The method for distinguishing the production of a cluster ion, $(A B)_{n}^{+}$, from either the parent $(A B) n$, or the higher order cluster, say $(A B)_{n+1}$, by dissociative ionization is based on the kinetic energy of the ion. Cold molecules and clusters produced in a skimmed molecular beam have exceedingly low translational energies in a direction perpendicular to the molecular beam axis. Thus, the ion time of flight distribution of an ion produced from such a cold precursor molecule is exceedingly narrow. On the other hand, 
dissociative ionization is invariably associated with the release of translational energy, which is often distributed isotropically. Thus, TOF peaks of such ions are broad. We have found that the TOF peak of an $(A B)_{n}^{+}$ cluster ion produced by dissociative ionization from a higher order neutral cluster is at least an order of magnitude broader than a similar mass ion produced by non-dissociative ionization.

Figure 1 shows the TOF distributions for acetylene ion monomers, dimers and trimers. The monomer ion signal is narrow. Its width of $13 \mathrm{~ns}$ indicates that the temperature of the molecular beam in direction perpendicular to the beam axis is about $4 \mathrm{~K}$. On the other hand, the dimer and trimer ion peaks are broad because of the translational energy released in the dissociative ionization of higher order clusters. The translational temperatures of the dimer and trimer ions, assuming Maxwell-Boltzmann distributions of translational velocities, are 80 and $100 \mathrm{~K}$, respectively. The Gaussian TOF distribution is, in fact, good evidence that the translational energy release is statistical and well described by a translational temperature.

The TOF spectra in Figure 1 indicate that no acetylene clusters are ionized directly to the corresponding ion. Rather, all are produced by dissociative ionization of higher order clusters. This is true even at the very lowest photon energies investigated.

Studies on several other systems, including ethylene clusters, methylchioride clusters, and methanol clusters indicate that essentially $100 \%$ of the cluster ions produced at or near the ionization onset come from dissociative ionization of higher order clusters. This means that determinations of, for instance, ionization energies of clusters by measuring the photoionization onset are unreliable. They represent only upper limits.

The non-observation of direct ionization of clusters lies in the structures of the neutral and ionic clusters, that is, in the Franck-Condon factors associated with the ionization process. This has been discussed by a number of workers. We have carried out, as others have, ab initio calculations on some of these clusters and their corresponding ions. In the case of acetylene, we have found that the trimer ion is totaliy unstable when we begin with a geometry corresponding to that of the neutral. The ion dissociates to a dimer ion, which has a structure that is neither dimer like, nor like any other $\mathrm{C}_{4} \mathrm{H}_{4}^{+}$ion known so far.

Other studies in our laboratory are dealing with the role of rotational energy in the dissociation process. The cold samples make possible the study of rotational effects in dissociation reactions. The dissociation onset for a reaction is shifted to higher energies when the molecule is cooled to $0 \mathrm{~K}$. The shift is equal to the internal vibrational energy plus any rotational energy that can be used to overcome the dissociation onset. Loose and tight transition states are thought to differ in the number of rotational degrees of freedom that can contribute. However, quantitative data for this effect are lacking. 


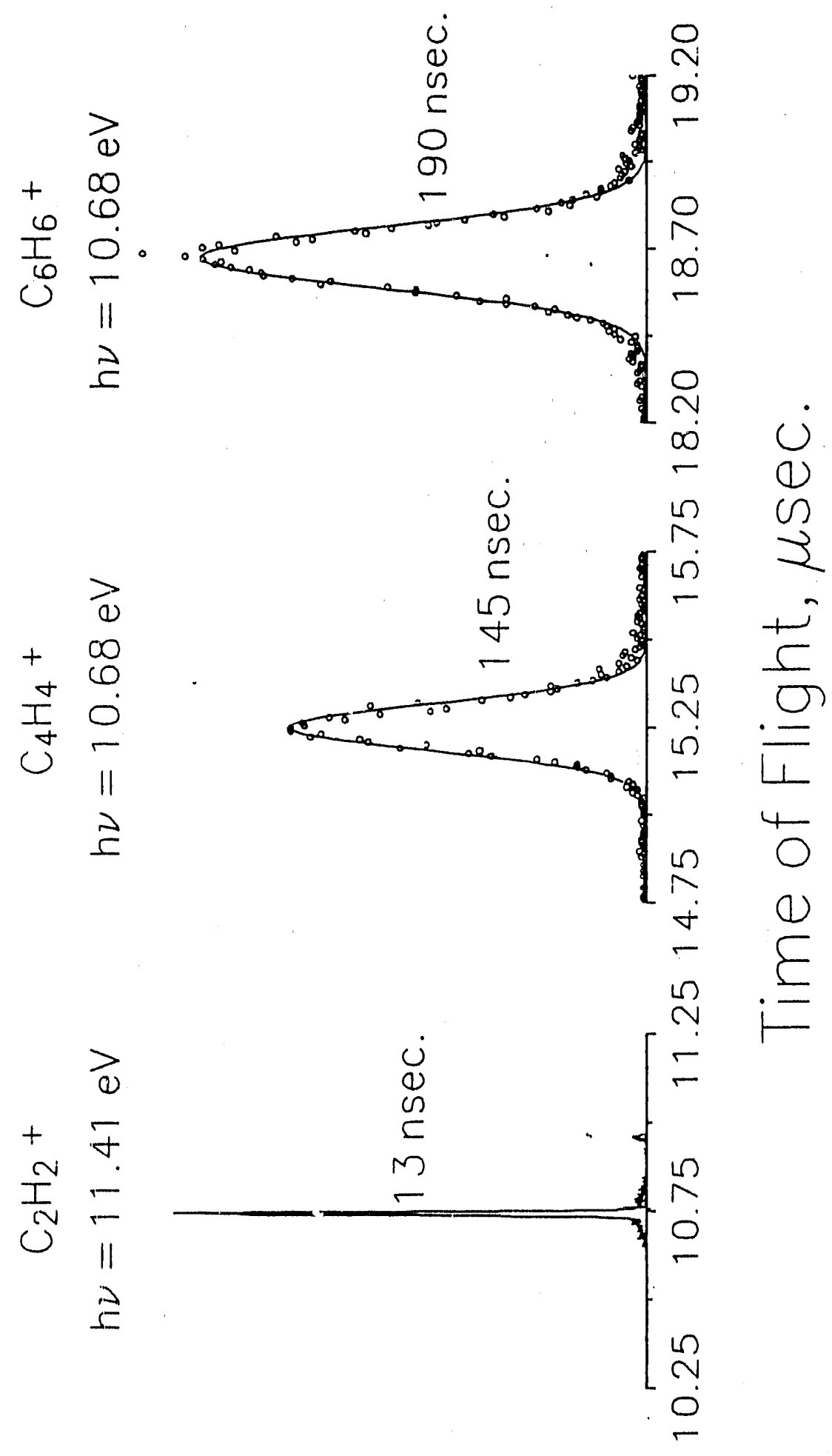

Figure 1 
Research Publications 1990, 1991, 1992:

J.S. Riley, T. Baer and G.D. Marbury, "Sequential Ortho Effects: Characterization on Novel $[M-35]^{+}$Fragment Ions in the Mass Spectra of 2alky1-4,6-Dinitrophenols," J. Am. Soc. Mass Spectrom. 269 (1991).

K.M. Weitze1, J. Booze and T. Baer, "Shifts in Photoionization Fragmentation Onsets; A Direct Measure of Cooling in a Supersonic Molecular Beam," Chem. Phys. 150263 (1991).

T. Baer, K.M. Weitzel and J. Booze, "Photoelectron Photoion Coincidence Studies of Ion Dissociation Dynamics" in Vacuum U1traviolet Ionization and Dissociation of Molecules and Clusters, World Scientific, Inc., C.Y. Ng, Ed. (1991) p259-296.

T. Baer, "The Measurement and Interpretation of Onset Energies," NATO ASI Series, K. Jennings, Ed. (1991).

J.A. Booze, K.M. Weitzel and T. Baer, "The Rates of $\mathrm{HCl}$ Loss from Energy Selected Ethylchloride Ions: A Case of Tunneling Through an H-Atom Transfer Barrier," J. Chem. Phys. 94 3649-3656 (1991).

K.M. Weitze1, J.A. Booze and T. Baer, "TPEPICO Study of the Ethane Loss from Energy Selected $n$-Pentane Ions Cooled in a Supersonic Expansion," Int. J. Mass Spectrom. Ion Proc. 107301 (1991).

K.M. Weitze1, J.A. Booze and T. Baer, "The Metastable Formation of diethylchloronium Ions from Ethylchloride Dimers in a Seeded Molecular Beam, " $Z$. Phys. D. 18383 (1991).

J. Riley and T. Baer, "Dissociation Dynamics of Phenetole Cations by Photoelectron Photoion Coincidence," J. Am. Soc. Mass Spectrom. 2464 (1991).

0 . Dutuit and T. Baer, "Isotope Effect in the Dissociation of Partially Deuterated Dimethyl Ether, $\mathrm{CH}_{3} \mathrm{OCD}_{3}{ }^{+}$Ions," Int. J. Mass Spectrom. Ion Proc. 11067 (1991).

T. Baer and J.A. Borze, "Long Lived Ion Complexes," in Ion-Molecule Collision Complexes, W. Hase Ed. JAI Press (1992), in press.

J.A. Booze and T. Baer, "On the Determination of Cluster Properties by Ionization Techniques," J. Chem. Phys. (in press).

J.A. Booze and T. Baer, "Ab initio Study of $\mathrm{C}_{3} \mathrm{H}_{8} \mathrm{O}^{+}$Ions," J. Phys. Chem. (in press).

J.A. Booze and T. Baer, "Dissociation Dynamics of Energy Selected $\mathrm{CH}_{3} \mathrm{CH}_{2} \mathrm{CH}_{2} \mathrm{OH}^{+}$ and $\mathrm{CH}_{3} \mathrm{CH}_{2} \mathrm{CH}_{2} \mathrm{OH}^{+}$Ions," J. Phys. Chem. (in press). 

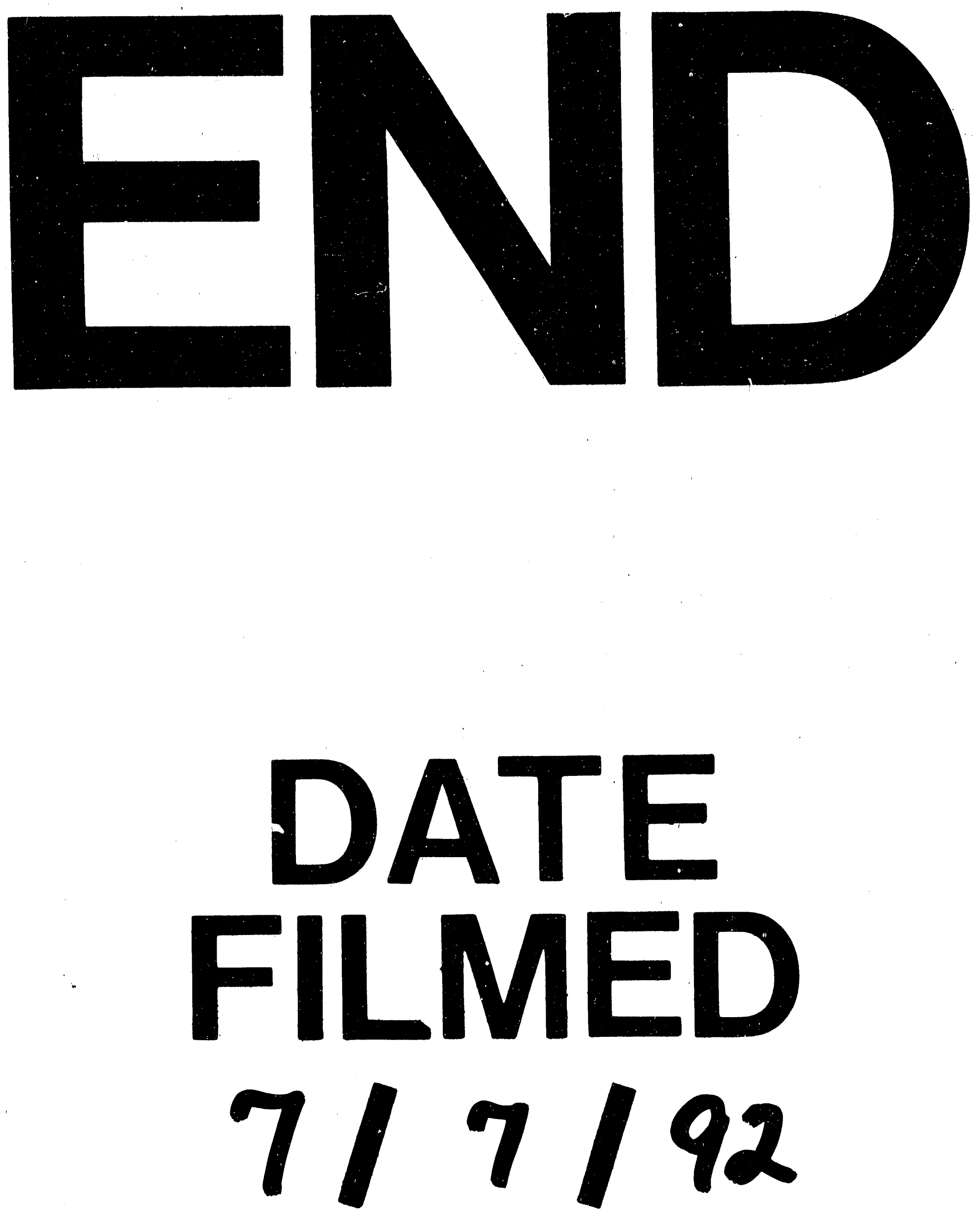
